Göteborg ITP 98-2, DAMTP-98-21, HKS-NT-FR-98/2-SE

31 March 1998

(Revised 22 March 1999)

\title{
A general BRST approach to string theories with zeta function regularizations
}

\author{
Stephen Hwang円, \\ Karlstad University \\ S-65188 Karlstad, Sweden \\ Robert Marnelius?2, \\ ITP, Chalmers University of Technology \\ Göteborg University \\ S-412 96 Göteborg, Sweden \\ Panagiotis Saltsidis \\ DAMTP, University of Cambridge \\ Silver St, Cambridge CB3 9EW, UK
}

\begin{abstract}
We propose a new general BRST approach to string and string-like theories which have a wider range of applicability than e.g. the conventional conformal field theory method. The method involves a simple general regularization of all basic commutators which makes all divergent sums to be expressible in terms of zeta functions from which finite values then may be extracted in a rigorous manner. The method is particular useful in order to investigate possible state space representations to a given model. The method is applied to three string models: The ordinary bosonic string, the tensionless string and the conformal tensionless string. We also investigate different state spaces for these models. The tensionless string models are treated in details. Although we mostly rederive known results they appear in a new fashion which deepens our understanding of these models. Furthermore, we believe that our treatment is more rigorous than most of the previous ones. In the case of the conformal tensionless string we find a new solution for $d=4$.
\end{abstract}

\footnotetext{
${ }^{1}$ E-mail: Stephen.Hwang@hks.se

${ }^{2}$ E-mail: tferm@fy.chalmers.se

${ }^{3}$ E-mail: P.Saltsidis@damtp.cam.ac.uk
} 


\section{Introduction and presentation of the method.}

The usual operator formulation of BRST quantization of string theories are based on the following ingredients: First specify a BRST invariant vacuum state and then normal order the BRST operator in order to finally check in which dimension the BRST operator is nilpotent [1]. Even the conformal field theory method is based on these ingredients [2]. These methods have been very successful when applied to conventional string theories. However, there are models to which these methods are not applicable. We have e.g. the tensionless string models which do not have a conventional vacuum state [3, 何. In this paper we present a new rigorous and very general approach to the operator version of BRST quantization, which not only makes it possible to consistently treat the conventional models but also the tensionless string models. The method is particularly useful in order to investigate possible state space representations to a given model. The method seems, furthermore, to cast new light on the BRST method as a whole.

What we advocate here is the following general procedure in the BRST quantization:

\section{Construct a hermitian and formally nilpotent BRST operator $Q$.}

2. Find a state space such that the properties above are true as operator equations in this space and for which the equation $Q|\phi\rangle=0$ has non-trivial solutions.

3. Use a general precise regularization of the basic commutators in the above analysis.

The idea to start with a nilpotent BRST operator and then look for possible solutions is of course very natural. However, usually one prescribes the setting first, which is what mathematicians would tell us to do. Usually the state space governs how we construct the BRST charge. But here this is not required. We only require a formally nilpotent BRST charge to start with. Then we look for possible state spaces. This freedom seems quite large. However, it is what a physicist likes to play with since physical models should also allow for our physical intuition to act. The structure of possible solutions of such a general prescription for finite degrees of freedom was given in [0] and further developed in [6]. One may note that a hermitian, nilpotent BRST charge may be constructed for a very large class of models in terms of a power expansion in $\hbar$ and in the ghost fields [7]. A solution is naturally obtained in a Weyl ordered form. However, these properties are only formal, since we must also find a state space in which the BRST charge makes sense. In order to do this in the case of infinite degrees of freedom we must make use of a regularization procedure. For instance, in the string models we encounter divergent sums. Such sums must be regularized in some way. Here we consider a simple general regularization of the basic commutators, a regularization which will make all infinite sums to be expressible as zeta functions. This regularization, which is presented in section 2, is therefore such that when the regulator is removed it will give finite results through analytic continuation in all cases considered. This makes it possible to rigorously compute all operator equations. Furthermore, and which is important, it allows us to investigate possible state spaces both rigorously and efficiently. We emphasize that the zeta function regularization here is used in a much more general and precise form than what one usually finds since it here appears from one single regularization of the basic commutators. (For applications of zeta regularization and for literature on the subject, see e.g. [8].) 
Instead of giving a detailed prescription how the method is supposed to be applied, we treat three string models in details: the closed ordinary bosonic string, the closed tensionless string and the closed conformal tensionless string. The closed ordinary bosonic string is mainly treated for pedagogical reasons. However, it should be interesting to see how we investigate alternative state space representations. Our main interest is in the tensionless strings. Here we give a rather exhaustive analysis from which we are able to give precise results for all proposed versions.

Critical dimensions in string theories appear in connection with a particular state space. In our method they appear through an inconsistency: The formally nilpotent BRST charge is not nilpotent or is not appropriate on the considered state space. In our investigation of three string models we first look for a BRST invariant vacuum state. Then we check whether or not the formally nilpotent BRST charge is nilpotent on this vacuum state. The conventional closed bosonic string turns out to be the most intricate example here. The conventional vacuum state is investigated in section 3. The appropriate BRST charge turns out to be the formally nilpotent charge shifted by a regulator dependent term (eq.(3.22)). This charge is, however, only nilpotent at $d=26$. Thus, the standard results are obtained but in a different way than usually. This treatment demonstrates how our method works and maybe it also deepens our understanding of this well-known model. The second model we treat is the tensionless string [9, 19, 11]. In section 4 we recover the known result of no critical dimension in [10] for one vacuum state, and in section 5 we find the critical dimension 26 as was found in 11 for another vacuum state. Our final example is the conformal tensionless string. In section 6 we first investigate the state space considered in [4]. Here we find that the BRST charge is nilpotent in any dimension. However, the vacuum state is only BRST invariant in two dimensions and we have not found any BRST invariant states in other dimensions. In section 7 we also consider another state space, which to our knowledge has not been considered previously. In this state space the BRST charge is again nilpotent in arbitrary dimensions, but the vacuum state is only BRST invariant in $d=4$. For other values of $d$ we have not found any BRST invariant states in this state space. In section 7 we investigate also the alternative vacuum state considered in [3] in which case we find that the BRST charge is not nilpotent in any dimensions and, hence, that there is no consistent BRST treatment at all, which is in agreement with the result of [3]. In sections 8 and 9 we investigate alternative vacua also for the ordinary bosonic string. Note that unlike some previous calculations our regularization makes all results finite which makes us believe that the treatment given here is in general more rigorous than previous ones.

Normally a consistent BRST quantization requires BRST invariant states with positive norms. In fact, such a condition should really be inserted into the condition 2 above. However, this would then exclude all models we treat except possibly the ordinary bosonic string. The reason is that all our calculations are performed in the minimal sector which is the sector with no dynamical Lagrange multipliers and antighosts. An artefact of this sector is that one cannot in general work on a truly inner product space. Consistent BRST invariant states $|\phi\rangle$ requires here the existence of a dual BRST invariant state $|\bar{\phi}\rangle$ satisfying the condition [5]

$$
\langle\bar{\phi} \mid \phi\rangle \text { is finite and } \neq 0 \text {. }
$$

This condition restricts the possible solutions. To work on truly inner product spaces one has to consider a BRST quantization in the complete sector with dynamical Lagrange 
multipliers and antighosts [12]. However, inconsistent solutions in the minimal sector will remain inconsistent in the complete sector. Furthermore, solutions containing BRST invariant negative norm states in the minimal sector will retain these in the complete sector. In section 9 we present a state space representation for the conventional bosonic string model which yields a finite set of BRST invariant states, which however are shown to contain negative norm states. The same is true for the special solutions of the tensionless string in section 5. Both these options have therefore to be excluded. The consistency of the tensionless string model in section 4 seems unclear, since it has a vacuum solution which is not associated with any oscillators. The BRST invariant states are not inner product states at all in the minimal sector. Although we expect there exist a positive normed solution in the complete sector, this remains to be investigated.

It is of course also possible to investigate the fermionic extensions of the models considered here [11, 13]. We expect such calculations to be quite straightforward using the method we present here. One may also consider other theories like e.g. brane-theories [14] and conventional field theories. We believe that also here our method should be important for rigorous results.

\section{Our regularization}

In string theory the string coordinates $X^{\mu}(\tau, \sigma)$ and the corresponding conjugate momenta $P_{\mu}(\tau, \sigma)$ satisfy the basic nonzero equal-time commutator (we suppress $\tau$ in the following)

$$
\left[X^{\mu}(\sigma), P_{\nu}\left(\sigma^{\prime}\right)\right]=i \delta_{\nu}^{\mu} \delta\left(\sigma-\sigma^{\prime}\right)
$$

Since we shall only consider closed strings we let $X^{\mu}(\sigma)$ and $P_{\mu}(\sigma)$ be periodic functions with period $\pi$. Thus, we may set $\left(\sum_{n} \equiv \sum_{n=-\infty}^{\infty}\right.$ in the following)

$$
X^{\mu}(\sigma)=\frac{1}{\sqrt{\pi}} \sum_{n} x_{n}^{\mu} e^{-2 i n \sigma}, \quad P^{\mu}(\sigma)=\frac{1}{\sqrt{\pi}} \sum_{n} p_{n}^{\mu} e^{-2 i n \sigma}
$$

and replace (2.1) by

$$
\left[x_{n}^{\mu}, p_{m \nu}\right]=i \delta_{\nu}^{\mu} \delta_{n+m}^{0} .
$$

The delta function in (2.1) is then the periodic delta function

$$
\delta\left(\sigma-\sigma^{\prime}\right)=\frac{1}{\pi} \sum_{n} e^{2 i n\left(\sigma-\sigma^{\prime}\right)} .
$$

It is the appearance of a delta function in the commutator (2.1) that causes infinities in the quantum string theory. In order to handle these infinities in a well defined manner we have to regularize the delta function, i.e. we have to make it an ordinary well defined function. We have therefore to consider a modified or regularized commutator. We choose it to be of the form

$$
\left[x_{n}^{\mu}, p_{m \nu}\right](s)=i \delta_{\nu}^{\mu} \delta_{n+m}^{0} f(|n|, s),
$$

where $f(|n|, s)$ is a real function which satisfies the condition

$$
f(|n|, 0)=1 .
$$


It can only depend on the absolute value of $n$ in order for $x_{n}$ and $p_{n}$ to retain their hermiticity properties from (2.2) even in the regularized case, i.e. $\left(x_{n}\right)^{\dagger}=x_{-n}$ and $\left(p_{n}\right)^{\dagger}=$ $p_{-n}$. The choice of regulator function $f(|n|, s)$ is dictated by two considerations. Firstly, in the computations that will follow e.g. a calculation of the BRST nilpotency, the regulator should lead to a finite result as the regulator is removed $(s \rightarrow 0)$. Secondly, we would like our regulator to be as general as possible. A precise choice of $f(|n|, s)$ which satisfies these criteria is

$$
f^{(\alpha)}(|n|, s)= \begin{cases}(|n|+\alpha)^{-s}, & |n| \geq-A+1 \\ 1, & |n| \leq-A\end{cases}
$$

where $\alpha$ is a real constant, which may be chosen to have any value, and where $A$ is the closest integer to $\alpha$ satisfying $A \geq \alpha$. This choice will make all infinite sums to be expressible in terms of zeta functions (see below). This in turn will allow us to rigorously treat all infinite sums. Other regularizations are of course possible to use and should yield equivalent results. However, we think that the choice (2.7) is the most general one.

In the following BRST treatment of the various string models we have also the fermionic ghost variable $c^{I}(\sigma)$ and $b^{I}(\sigma)$. Their basic commutators must be regularized in exactly the same way as in (2.5). For ghosts $c_{n}^{I}, b_{n}^{I}$ (where $I$ label different types of ghosts) we have :

$$
\left[b_{m}^{I}, c_{n}^{J}\right](s)=\delta_{m+n}^{0} \delta^{I J} f^{(\alpha)}(|n|, s)
$$

The same is true for all other canonical variables one introduces. (We use graded commutators throughout.)

When calculating commutators we often get infinite sums of the form $\sum_{n} f^{(\alpha)}(|n|, s)$ which converge for $s>1$. However, by analytic continuations they may yield a finite value for $s<1$ and in particular for $s=0$. The choice (2.7) makes these sums expressible in terms of zeta functions. We have

$$
\sum_{n} f^{(\alpha)}(|n|, s)= \begin{cases}1-2 A+2 \zeta(s \mid 1+\alpha-A), & \alpha \leq 0 \\ -\alpha^{-s}-2 \sum_{n=0}^{A-2}(n+1+\alpha-A)^{-s}+2 \zeta(s \mid 1+\alpha-A), & \alpha>0\end{cases}
$$

where $\zeta(s \mid a)$ is the Hurwitz zeta function defined for $0<a \leq 1, s>1$ by [15]

$$
\zeta(s \mid a) \equiv \sum_{n=0}^{\infty} \frac{1}{(n+a)^{s}}
$$

By analytic continuation one finds the following value at $s=0(0<a \leq 1)$ 15

$$
\zeta(0 \mid a)=\frac{1}{2}-a
$$

which implies that (notice that the finite sums for $\alpha \leq 0$ and $\alpha>0$ are equal for $s=0$ in $(2.9))$

$$
\sum_{n} f^{(\alpha)}(|n|, 0)=-2 \alpha
$$


We have similarly e.g. (we set $s=0$ for the finite sums)

$$
\begin{aligned}
\sum_{n=1}^{\infty} n f^{(\alpha)}(|n|, s)= & \zeta(s-1 \mid 1+\alpha-A)-\alpha \zeta(s \mid 1+\alpha-A)+\frac{1}{2} A(A-1) \\
\sum_{n=1}^{\infty} n^{2} f^{(\alpha)}(|n|, s)=\quad & \zeta(s-2 \mid 1+\alpha-A)-2 \alpha \zeta(s-1 \mid 1+\alpha-A)+\alpha^{2} \zeta(s \mid 1+\alpha-A)- \\
& -\frac{1}{6} A(A-1)(2 A-1),
\end{aligned}
$$

which implies

$$
\lim _{s \rightarrow 0} \sum_{n=1}^{\infty} n f^{(\alpha)}(|n|, s)=\frac{1}{2} \alpha^{2}-\frac{1}{12}, \quad \lim _{s \rightarrow 0} \sum_{n=1}^{\infty} n^{2} f^{(\alpha)}(|n|, s)=-\frac{1}{3} \alpha^{3},
$$

since $\zeta(-1 \mid a)=\left(6 a-6 a^{2}-1\right) / 12$ and $\zeta(-2 \mid a)=a\left(3 a-2 a^{2}-1\right) / 6$.

The regularization (2.5) and (2.7) correspond by (2.1) to the following regularized delta function

$$
\delta_{s}\left(\sigma-\sigma^{\prime}\right)=\frac{1}{\pi}\left\{\sum_{n} f^{(\alpha)}(|n|, s) e^{2 i n\left(\sigma-\sigma^{\prime}\right)}\right\},
$$

which is a well defined function for $s>1$ and by eq. (2.12) gives a regularization such that $\lim _{s \rightarrow 0} \delta_{s}(0)=\frac{-2 \alpha}{\pi}$. Note here that depending on the value of $\alpha$ we can get any value in this limit. This is one argument supporting our belief that our regularization gives

the most general result possible. Notice also that $\lim _{s \rightarrow 0} \delta_{s}^{(k)}(0)$ is finite for any order of derivative $k$.

\section{$3 \quad$ The bosonic string}

As an illustration of our method we first treat the ordinary bosonic string. The bosonic string is classically characterized by the constraints

$$
\frac{1}{4 T}\left(P+T X^{\prime}\right)^{2}(\sigma)=0, \quad \frac{1}{4 T}\left(P-T X^{\prime}\right)^{2}(\sigma)=0,
$$

where $T$ is the string tension. The Fourier modes of the corresponding hermitian constraint operators are

$$
L_{n} \equiv \frac{1}{2} \sum_{k} \alpha_{n-k} \cdot \alpha_{k}, \quad K_{n} \equiv \frac{1}{2} \sum_{k} \tilde{\alpha}_{n-k} \cdot \tilde{\alpha}_{k}
$$

where

$$
\begin{aligned}
\alpha_{n}^{\mu} & \equiv\left(\frac{1}{2 \sqrt{T}} p_{n}^{\mu}-i \sqrt{T} n x_{n}^{\mu}\right), \\
\tilde{\alpha}_{n}^{\mu} & \equiv\left(\frac{1}{2 \sqrt{T}} p_{-n}^{\mu}-i \sqrt{T} n x_{-n}^{\mu}\right),
\end{aligned}
$$


in terms of the $x_{n}$ and $p_{n}$ modes in (2.2). The regularized commutator (2.5) with the regularized function (2.7) imply

$$
\begin{aligned}
& {\left[\alpha_{n}^{\mu}, \alpha_{m}^{\nu}\right](s)=n \eta^{\mu \nu} f^{(\alpha)}(|n|, s) \delta_{n+m}^{0}, \quad\left[\alpha_{n}^{\mu}, \tilde{\alpha}_{m}^{\nu}\right](s)=0,} \\
& {\left[\tilde{\alpha}_{n}^{\mu}, \tilde{\alpha}_{m}^{\nu}\right](s)=n \eta^{\mu \nu} f^{(\alpha)}(|n|, s) \delta_{n+m}^{0},}
\end{aligned}
$$

where $\eta^{\mu \nu}$ is a Minkowski metric, $\operatorname{diag}\left(\eta^{\mu \nu}\right)=(-1,+1,+1,+1)$. We consider now a corresponding BRST theory in which the manifestly hermitian BRST charge is given by

$$
\begin{aligned}
Q= & \sum_{k}\left(L_{-k} c_{k}^{L}+K_{-k} c_{k}^{K}\right)+ \\
& +\frac{1}{4} \sum_{k, l}(k-l)\left(b_{k+l}^{L} c_{-l}^{L} c_{-k}^{L}+b_{k+l}^{K} c_{-l}^{K} c_{-k}^{K}-c_{-k}^{L} c_{-l}^{L} b_{k+l}^{L}-c_{-k}^{K} c_{-l}^{K} b_{k+l}^{K}\right),
\end{aligned}
$$

where $c_{k}^{L}, b_{k}^{L}$ and $c_{k}^{K}, b_{k}^{K}$ are fermionic ghost modes satisfying (2.8). In the $s \rightarrow 0$ limit this BRST charge is formally nilpotent. A consequence of this nilpotency is that the extended constraints, $\left[Q, b_{k}^{I}\right]$, satisfy a closed algebra without any central extensions. The extended constraints to $L_{n}$ and $K_{n}$ are

$$
\begin{aligned}
& \tilde{L}_{n} \equiv\left[Q, b_{n}^{L}\right]=L_{n}-\frac{1}{2} \sum_{k}(k+n)\left(c_{k}^{L} b_{n-k}^{L}-b_{n-k}^{L} c_{k}^{L}\right), \\
& \tilde{K}_{n} \equiv\left[Q, b_{n}^{K}\right]=K_{n}-\frac{1}{2} \sum_{k}(k+n)\left(c_{k}^{K} b_{n-k}^{K}-b_{n-k}^{K} c_{k}^{K}\right) .
\end{aligned}
$$

For non-zero $s$ we have e.g. the following crucial commutator

$$
\begin{aligned}
& {\left[\tilde{L}_{m}, \tilde{L}_{-m}\right](s)=} \\
& \frac{1}{2} m \sum_{k}\left(\alpha_{-k} \cdot \alpha_{k}+k\left(c_{-k}^{L} b_{k}^{L}-b_{k}^{L} c_{-k}^{L}\right)\right)\left(f^{(\alpha)}(|m+k|, s)+f^{(\alpha)}(|m-k|, s)\right)+ \\
& +\frac{1}{2} \sum_{k}\left(k \alpha_{-k} \cdot \alpha_{k}+\left(2 m^{2}-k^{2}\right)\left(b_{k}^{L} c_{-k}^{L}-c_{-k}^{L} b_{k}^{L}\right)\right)\left(f^{(\alpha)}(|m+k|, s)-f^{(\alpha)}(|m-k|, s)\right),
\end{aligned}
$$

which in the limit $s \rightarrow 0$ becomes

$$
\left[\tilde{L}_{m}, \tilde{L}_{-m}\right](0)=2 m \tilde{L}_{0}
$$

which is consistent with the fact that the BRST charge (3.5) is nilpotent in the $s \rightarrow 0$ limit. Now the crucial point is that the $s \rightarrow 0$ limit has no meaning before we specify on which state space the operators act. Below we show that the conventional choice of state space imply the expected result that we have a nilpotent BRST charge only in spacetime dimensions $d=26$ in the $s \rightarrow 0$ limit.

The standard choice of a vacuum state, $|0\rangle$, satisfies

$$
\alpha_{m}|0\rangle=\tilde{\alpha}_{m}|0\rangle=0, \quad \forall m>0 .
$$

In order for this vacuum to be BRST invariant, $Q|0\rangle=0$, we must require the consistency conditions

$$
\left[Q, \alpha_{m}^{\mu}\right]|0\rangle=0 \quad \Rightarrow m f^{(\alpha)}(|m|, s) \sum_{k=m}^{\infty} c_{k}^{L} \alpha_{m-k}^{\mu}|0\rangle=0
$$




$$
\begin{aligned}
& \Rightarrow c_{m}^{L}|0\rangle=0, \quad \forall m>0, \\
{\left[Q, \tilde{\alpha}_{m}^{\mu}\right]|0\rangle=0 \quad } & \Rightarrow m f^{(\alpha)}(|m|, s) \sum_{k=m}^{\infty} c_{k}^{K} \tilde{\alpha}_{m+k}^{\mu}|0\rangle=0 \\
& \Rightarrow c_{m}^{K}|0\rangle=0, \quad \forall m>0 .
\end{aligned}
$$

These conditions in turn allow for the additional conditions

$$
b_{m}^{L}|0\rangle=b_{m}^{K}|0\rangle=0, \quad \forall m \geq 0,
$$

for which the consistency conditions are

$$
\begin{array}{ll}
{\left[Q, b_{m}^{L}\right]|0\rangle \equiv \tilde{L}_{m}|0\rangle=0, \quad \forall m \geq 0,} \\
{\left[Q, b_{m}^{K}\right]|0\rangle \equiv \tilde{K}_{m}|0\rangle=0, \quad \forall m \geq 0 .}
\end{array}
$$

They are satisfied for $m>0$, but for $m=0$ the situation is unclear. For non-zero $s$ we have

$$
\tilde{L}_{0}(s)|0\rangle=\frac{1}{2}\left(\alpha_{0}^{2}+(d-2) \sum_{k=1}^{\infty} k f^{(\alpha)}(|k|, s)\right)|0\rangle,
$$

which in the $s \rightarrow 0$ limit leads to the following finite expression

$$
\tilde{L}_{0}|0\rangle=\frac{1}{2}\left(\alpha_{0}^{2}+(d-2)\left(\frac{1}{2} \alpha^{2}-\frac{1}{12}\right)\right)|0\rangle,
$$

where we have made use of the relation (2.14). The conditions (3.13) with the property (3.15) and a similar one for $\tilde{K}_{n}$ require the vacuum state to be an eigenstate to the momentum operator $p_{0}^{\mu}$ with an eigenvalue which depends on the parameter $\alpha$ in the regularization function (2.7). (Notice that (3.3) implies $\alpha_{0}^{2}=\tilde{\alpha}_{0}^{2}=p_{0}^{2} / 4 T$.) This is an unsatisfactory result. It means that the conventional vacuum state is not BRST invariant under the formally nilpotent BRST charge above. In fact, $Q$ is not even nilpotent on the conventional vacuum state.

That the BRST operator (3.5) is not nilpotent on the above vacuum state may be seen by calculating the commutator $\left[\tilde{L}_{m}, \tilde{L}_{-m}\right]$ on the vacuum state for non-zero $s$. We find from eq. (3.8)

$$
\begin{aligned}
{\left[\tilde{L}_{m}, \tilde{L}_{-m}\right](s)|0\rangle=} & \left(m f^{(\alpha)}(|m|, s) \alpha_{0}^{2}+\frac{1}{2} d \sum_{k=1}^{m} k(m-k) f^{(\alpha)}(|k|, s) f^{(\alpha)}(|k-m|, s)-\right. \\
& \left.-\sum_{k=1}^{m}(2 m-k)(k+m) f^{(\alpha)}(|k|, s) f^{(\alpha)}(|k-m|, s)\right)|0\rangle
\end{aligned}
$$

In the $s \rightarrow 0$ limit the right-hand side becomes

$$
\left(m \alpha_{0}^{2}+\frac{1}{12}(d-26) m^{3}-\frac{1}{12}(d-2) m\right)|0\rangle,
$$

which only is zero if $d=26$ and if the eigenvalue of $\alpha_{0}^{2}$ is 2 , which is a regulator independent condition. The reason for the different results from (3.9) and (3.15) is due to the fact that on the right-hand side of (3.8) the factor $f^{(\alpha)}(|m+k|, s)-f^{(\alpha)}(|m-k|, s)$, which is zero in 
the $s \rightarrow 0$ limit, is multiplied by an operator which is infinite on the above vacuum state. This means that $\tilde{L}_{0}$ and $\tilde{K}_{0}$ are not zero on the vacuum state $|0\rangle$ when (3.17) is zero. The solution of this dilemma is found when we rewrite the right-hand side of the commutator (3.8) by means of the regularized commutators (3.4) as follows

$$
\begin{aligned}
& {\left[\tilde{L}_{m}, \tilde{L}_{-m}\right](s)=} \\
& m \sum_{k=1}^{\infty}\left(\alpha_{-k} \cdot \alpha_{k}+k b_{-k}^{L} c_{k}^{L}+k c_{-k}^{L} b_{k}^{L}\right)\left(f^{(\alpha)}(|m+k|, s)+f^{(\alpha)}(|m-k|, s)\right)+ \\
& +\sum_{k=1}^{\infty}\left(k \alpha_{-k} \cdot \alpha_{k}+\left(k^{2}-2 m^{2}\right)\left(b_{-k}^{L} c_{k}^{L}+c_{-k}^{L} b_{k}^{L}\right)\right)\left(f^{(\alpha)}(|m+k|, s)-f^{(\alpha)}(|m-k|, s)\right)+ \\
& +m f^{(\alpha)}(|m|, s) \alpha_{0}^{2}+\frac{1}{2} d \sum_{k=1}^{m} k(m-k) f^{(\alpha)}(|k|, s) f^{(\alpha)}(|k-m|, s)- \\
& -\sum_{k=1}^{m}(2 m-k)(k+m) f^{(\alpha)}(|k|, s) f^{(\alpha)}(|k-m|, s) .
\end{aligned}
$$

This expression implies

$$
\begin{aligned}
{\left[\tilde{L}_{m}, \tilde{L}_{-m}\right]=} & 2 m\left(\frac{1}{2} \alpha_{0}^{2}+\sum_{k=1}^{\infty}\left(\alpha_{-k} \cdot \alpha_{k}+k b_{-k}^{L} c_{k}^{L}+k c_{-k}^{L} b_{k}^{L}\right)\right)+ \\
& +\frac{1}{12}(d-26) m^{3}-\frac{1}{12}(d-2) m
\end{aligned}
$$

in the $s \rightarrow 0$ limit in agreement with (3.16) and (3.17). This may be rewritten as follows

$$
\left[\tilde{L}_{m}, \tilde{L}_{-m}\right]=2 m \tilde{L}_{0}+\frac{1}{12}(d-26) m^{3}-\frac{1}{2} m(d-2) \alpha^{2},
$$

where

$$
\tilde{L}_{0}=\frac{1}{2} \alpha_{0}^{2}+\sum_{k=1}^{\infty}\left(\alpha_{-k} \cdot \alpha_{k}+k b_{-k}^{L} c_{k}^{L}+k c_{-k}^{L} b_{k}^{L}\right)+\frac{1}{2}(d-2)\left(\frac{1}{2} \alpha^{2}-\frac{1}{12}\right)
$$

is the original $\tilde{L}_{0}$ rewritten for finite $s$ and taking the limit $s \rightarrow 0$. Eq. (3.20) demonstrates the inconsistency with a nilpotent BRST charge obtained above and the reason why the conventional vacuum state is not BRST invariant. The remedy is obvious and expected: first we notice that we may get a closed algebra at $d=26$ if we redefine the extended constraint $\tilde{L}_{0}$. This in turn may be accomplished by a redefinition of the original BRST charge: Simply replace $Q$ in (3.5) by

$$
Q^{\prime}=Q-\frac{d-2}{4} \alpha^{2}\left(c_{0}^{L}+c_{0}^{K}\right)
$$

which is not formally nilpotent for $d \neq 2$. The corresponding extended constraints are

$$
\tilde{L}_{m}^{\prime}=\left[Q^{\prime}, b_{m}^{L}\right]=\left\{\begin{array}{l}
\tilde{L}_{0}-\frac{1}{4}(d-2) \alpha^{2}, m=0 \\
\tilde{L}_{m}, m \neq 0
\end{array} .\right.
$$

Note that $\tilde{L}_{0}^{\prime}$ in distinction to $\tilde{L}_{0}$ in 3.21 is independent of the regulator parameter $\alpha$. We have

$$
\tilde{L}_{0}^{\prime}=\frac{1}{2} \alpha_{0}^{2}+\sum_{k=1}^{\infty}\left(\alpha_{-k} \cdot \alpha_{k}+k b_{-k}^{L} c_{k}^{L}+k c_{-k}^{L} b_{k}^{L}\right)-\frac{(d-2)}{24}
$$


The spectrum is therefore regulator independent as it should be. For $d=26$ we find now from 3.20

$$
\left[\tilde{L}_{m}^{\prime}, \tilde{L}_{-m}^{\prime}\right]=2 m \tilde{L}_{0}^{\prime}
$$

which is consistent with a nilpotent $Q^{\prime}$ for $d=26$, and that the conventional BRST vacuum is BRST invariant under $Q^{\prime}$.

In sections 8 and 9 we investigate other vacuum states for the bosonic string theory.

\section{The bosonic tensionless string}

The bosonic tensionless string (see [9]) is characterized by the constraints

$$
P^{\mu}(\sigma) P_{\mu}(\sigma)=0, \quad P^{\mu}(\sigma) X_{\mu}^{\prime}(\sigma)=0 .
$$

These constraints follow from the bosonic string by dropping the term $T^{2}\left(X^{\prime}(\sigma)\right)^{2}$ which is assumed to be negligible in the $T \rightarrow 0$ limit. The Fourier modes of the corresponding hermitian constraint operators are

$$
\phi_{n}^{-1} \equiv \frac{1}{2} \sum_{k} p_{k} \cdot p_{n-k}, \quad \phi_{n}^{L} \equiv-i \frac{1}{2} \sum_{k} k\left(x_{k} \cdot p_{n-k}+p_{n-k} \cdot x_{k}\right) .
$$

A formally nilpotent BRST charge operator is here given by

$$
\begin{aligned}
Q= & \sum_{k}\left(\phi_{-k}^{-1} c_{k}^{-1}+\phi_{-k}^{L} c_{k}^{L}\right)-\frac{1}{2} \sum_{k, l}(k-l)\left(c_{-k}^{-1} c_{-l}^{L} b_{k+l}^{-1}+\right. \\
& \left.+b_{k+l}^{-1} c_{-k}^{-1} c_{-l}^{L}+\frac{1}{2} c_{-k}^{L} c_{-l}^{L} b_{k+l}^{L}+\frac{1}{2} b_{k+l}^{L} c_{-k}^{L} c_{-l}^{L}\right) .
\end{aligned}
$$

We can check the nilpotency of $Q$ by calculating the algebra of the extended constraints given by

$$
\begin{aligned}
& \tilde{\phi}_{n}^{-1} \equiv\left[Q, b_{n}^{-1}\right]=\phi_{n}^{-1}-\sum_{k}(n+k) c_{k}^{L} b_{n-k}^{-1} \\
& \tilde{\phi}_{n}^{L} \equiv\left[Q, b_{n}^{L}\right]=\phi_{n}^{L}-\frac{1}{2} \sum_{k}(k+n)\left(c_{k}^{-1} b_{n-k}^{-1}-b_{n-k}^{-1} c_{k}^{-1}+c_{k}^{L} b_{n-k}^{L}-b_{n-k}^{L} c_{k}^{L}\right) .
\end{aligned}
$$

A straight-forward calculation of the commutators yields

$$
\left[\tilde{\phi}_{m}^{-1}, \tilde{\phi}_{n}^{L}\right]=(m-n) \tilde{\phi}_{m+n}^{-1}, \quad\left[\tilde{\phi}_{m}^{L}, \tilde{\phi}_{n}^{L}\right]=(m-n) \tilde{\phi}_{m+n}^{L} .
$$

Hence, we conclude that the BRST charge (4.3) is nilpotent.

We now look for a possible BRST invariant vacuum state. Following [4, 10] we consider a vacuum state defined by

$$
p_{n}^{\mu}|0\rangle=b_{n}^{-1}|0\rangle=b_{n}^{L}|0\rangle=0, \quad \forall n .
$$

(The crucial part is the first conditions. They may be viewed as the $T \rightarrow 0$ limit of $(3.10)$ using (3.3).) In order for this vacuum state to be BRST invariant it has to satisfy the consistency conditions

$$
\left[Q, p_{n}^{\mu}\right]|0\rangle=\left[Q, b_{n}^{-1}\right]|0\rangle=\left[Q, b_{n}^{L}\right]|0\rangle=0, \quad \forall n,
$$


where

$$
\left[Q, p_{n}^{\mu}\right]=-n \sum_{k} p_{n-k}^{\mu} c_{k}^{L}
$$

The first two conditions and the last one for $n \neq 0$ are easily seen to be satisfied due to (4.6). The only nontrivial condition is the last one for $n=0$. However, we have

$$
\begin{aligned}
& \tilde{\phi}_{0}^{L}|0\rangle \equiv\left[Q, b_{0}^{L}\right]|0\rangle=\left(\phi_{0}^{L}-\frac{1}{2} \sum_{k} k\left[c_{k}^{-1} b_{-k}^{-1}-b_{-k}^{-1} c_{k}^{-1}+c_{k}^{L} b_{-k}^{L}-b_{-k}^{L} c_{k}^{L}\right]\right)|0\rangle= \\
& =-\frac{1}{2} \sum_{k} k p\left(i\left[p_{-k}^{\mu}, x_{k \mu}\right]-\left[b_{-k}^{-1}, c_{k}^{-1}\right]-\left[b_{-k}^{L}, c_{k}^{L}\right]\right)|0\rangle= \\
& =-\frac{1}{2}(d-2) \sum_{k} k f^{(\alpha)}(|k|, s)|0\rangle=0 .
\end{aligned}
$$

(It is zero for any choice of regulator function $f(|k|, s) !)$. It is then easily seen that

$$
Q|0\rangle=0
$$

which means that $|0\rangle$ defined by (4.6) is a BRST invariant vacuum state for any dimension $d$. We must finally check that the BRST charge is nilpotent on the vacuum state. This may be accomplished by checking the algebra of the extended constraints. The most non-trivial one is $\left[\tilde{\phi}_{m}^{L}, \tilde{\phi}_{-m}^{L}\right]$ for which we find

$$
\begin{aligned}
& {\left[\tilde{\phi}_{m}^{L}, \tilde{\phi}_{-m}^{L}\right](s)=\frac{i}{2} \sum_{k}(k+m) k f^{(\alpha)}(|k+m|, s)\left(x_{-k} \cdot p_{k}-x_{k} \cdot p_{-k}+\right.} \\
& \left.+p_{k} \cdot x_{-k}-p_{-k} \cdot x_{k}\right)+\frac{1}{2} \sum_{k}(k+2 m)(k-m) f^{(\alpha)}(|k+m|, s)\left(b_{-k}^{-1} c_{k}^{-1}-b_{k}^{-1} c_{-k}^{-1}+\right. \\
& \left.+c_{-k}^{-1} b_{k}^{-1}-c_{k}^{-1} b_{-k}^{-1}+b_{-k}^{L} c_{k}^{L}-b_{k}^{L} c_{-k}^{L}+c_{-k}^{L} b_{k}^{L}-c_{k}^{L} b_{-k}^{L}\right)
\end{aligned}
$$

A straightforward calculation yields that $\left[\tilde{\phi}_{m}^{L}, \tilde{\phi}_{-m}^{L}\right](0)|0\rangle$ is zero, which is consistent with eq. (4.9). To conclude, within our regularization scheme we have shown that there exists a BRST invariant vacuum state in any dimension. Furthermore, the BRST charge is nilpotent in the state space containing this vacuum state. This result is in agreement with the results of ref. [10]. Note that there exists a dual vacuum state $|\overline{0}\rangle$ satisfying $\langle\overline{0} \mid 0\rangle$ finite and different from zero. $|\overline{0}\rangle$ satisfies $x_{n}^{\mu}|\overline{0}\rangle=c_{n}^{-1}|\overline{0}\rangle=c_{n}^{L}|\overline{0}\rangle=0$ for all $n$ together with their consistency conditions.

\section{Alternative quantization of the bosonic tensionless string}

Instead of a vacuum state satisfying (4.6) we follow [11] and consider

$$
p_{m}^{\mu}|0\rangle=x_{m}^{\mu}|0\rangle=0, \quad m>0 .
$$

The consistency conditions are

$$
\left[Q, p_{m}^{\mu}\right]|0\rangle=\left[Q, x_{m}^{\mu}\right]|0\rangle=0, \quad m>0,
$$


and they require

$$
c_{m}^{-1}|0\rangle=c_{m}^{L}|0\rangle=0, \quad m>0
$$

for which

$$
\left[Q, c_{m}^{-1}\right]|0\rangle=\left[Q, c_{m}^{L}\right]|0\rangle=0, \quad m>0
$$

are automatically satisfied. This vacuum is then ghost fixed by

$$
b_{m}^{-1}|0\rangle=b_{m}^{L}|0\rangle=0, \quad m \geq 0 .
$$

The corresponding consistency conditions,

$$
\tilde{\phi}_{m}^{-1}|0\rangle=\tilde{\phi}_{m}^{L}|0\rangle=0, \quad m \geq 0
$$

are automatically satisfied for $m>0$. For $m=0$ we have

$$
\tilde{\phi}_{0}^{-1}|0\rangle=0 \quad \Leftrightarrow \quad p_{0}^{2}|0\rangle=0 .
$$

For such a condition to be meaningful the vacuum state should be an eigenstate to $p_{0}^{\mu}$, i.e. $p_{0}^{\mu}|p\rangle=p^{\mu}|p\rangle$. Eq.(5.7) requires then the vacuum to be massless $\left(p^{2}=0\right)$. For $m=0$ we have also

$$
\tilde{\phi}_{0}^{L}|p\rangle=(d-2)\left(\frac{1}{2} \alpha^{2}-\frac{1}{12}\right)|p\rangle
$$

which satisfies $(5.6)$ only for $d=2$. For $s \neq 0$ we have

$$
\begin{aligned}
& \tilde{\phi}_{0}^{L}(s)=i \sum_{k=1}^{\infty} k\left(x_{-k} \cdot p_{k}-p_{-k} \cdot x_{k}\right)+\sum_{k=1}^{\infty} k\left(b_{-k}^{-1} \cdot c_{k}^{-1}+c_{-k}^{-1} \cdot b_{k}^{-1}+b_{-k}^{L} \cdot c_{k}^{L}+\right. \\
& \left.+c_{-k}^{L} \cdot b_{k}^{L}\right)+(d-2) \sum_{k=1}^{\infty} k f^{(\alpha)}(|k|, s) .
\end{aligned}
$$

From the commutator (4.11) we find

$$
\lim _{s \rightarrow 0}\left[\tilde{\phi}_{m}^{L}, \tilde{\phi}_{-m}^{L}\right](s)|p\rangle=\frac{1}{6}\left((d-26) m^{3}-(d-2) m\right)|p\rangle .
$$

In fact, in the $s \rightarrow 0$ limit we have

$$
\left[\tilde{\phi}_{m}^{L}, \tilde{\phi}_{-m}^{L}\right]=2 m \tilde{\phi}_{0}^{L}-\frac{1}{2} \alpha^{2}(d-2) m+\frac{1}{6}(d-26) m^{3},
$$

where $\tilde{\phi}_{0}^{L}$ on the right-hand side is the $s \rightarrow 0$ limit of (5.9). If we redefine $Q$ by

$$
Q^{\prime} \equiv Q-\frac{1}{4} \alpha^{2}(d-2) c_{0}^{L}
$$

then the extended constraint operator $\tilde{\phi}_{0}^{L}$ is replaced by

$$
\tilde{\phi}_{0}^{L}=\tilde{\phi}_{0}^{L}-\frac{1}{4} \alpha^{2}(d-2) .
$$


This operator together with $\tilde{\phi}_{m}^{L}, m \neq 0$ satisfy then an anomaly free algebra for $d=26$ from which we conclude that $Q^{\prime}$ is nilpotent for $d=26$. However, from (5.8) we have

$$
\tilde{\phi}_{0}^{\prime L}|p\rangle=-\frac{1}{12}(d-2)|p\rangle .
$$

It follows that we have no BRST invariant vacuum of the above form for $d=26$. However, there are BRST invariant states in $d=26$. In [16] it was shown that there are massless states with spin 0,1 and 2 . In section 9 we show that these states do not have positive norms. (From the results of [11] it seems that a BRST invariant vacuum state only exists in the Ramond sector of the spinning tensionless string in which case the critical dimension is 10.)

\section{The bosonic conformal string}

The conformal string is a tensionless string which is made manifestly conformally invariant [3, 4]. By adding two extra dimensions, one timelike and one spacelike, one forms new coordinates that transforms as $S O(d, 2)$ vectors. By means of $S O(d, 2)$ invariant constraints one obtains then an $S O(d, 2)$ conformally invariant formulation by construction. Let $X^{M}=\left(X^{\mu}, X^{+}, X^{-}\right)$be the new coordinate vector where the metric of the new coordinates is $\eta_{++}=\eta_{--}=0, \eta_{+-}=\eta_{-+}=1$. Classically the constraints are

$$
\begin{aligned}
& \Phi^{-1}(\sigma) \equiv P^{M}(\sigma) P_{M}(\sigma)=0, \quad \Phi^{0}(\sigma) \equiv P^{M}(\sigma) X_{M}(\sigma)=0 \\
& \Phi^{1}(\sigma) \equiv X^{M}(\sigma) X_{M}(\sigma)=0, \quad \Phi^{L}(\sigma) \equiv P^{M}(\sigma) X_{M}^{\prime}(\sigma)=0
\end{aligned}
$$

and they reduce to the constraints (4.1) of the tensionless string by means of the gauge fixing conditions

$$
P^{+}(\sigma)=0, \quad X^{+}(\sigma)-1=0 .
$$

(A corresponding construction for particles were given in [17].) The hermitian BRST charge operator is given by

$$
\begin{aligned}
Q= & \sum_{k}\left(\phi_{-k}^{1} c_{k}^{1}+\phi_{-k}^{0} c_{k}^{0}+\phi_{-k}^{-1} c_{k}^{-1}+\phi_{-k}^{L} c_{k}^{L}\right) \\
& -\frac{1}{2} \sum_{k, l}\left(2 i c_{-k}^{1} c_{-l}^{-1} b_{k+l}^{0}+i c_{-k}^{1} c_{-l}^{0} b_{k+l}^{1}-i c_{-k}^{-1} c_{-l}^{0} b_{k+l}^{-1}\right. \\
& +(k+l) c_{-k}^{1} c_{-l}^{L} b_{k+l}^{1}+(k-l) c_{-k}^{-1} c_{-l}^{L} b_{k+l}^{-1}+k c_{-k}^{0} c_{-l}^{L} b_{k+l}^{0} \\
& \left.+\frac{1}{2}(k-l) c_{-k}^{L} c_{-l}^{L} b_{k+l}^{L}+\text { h.c. }\right),
\end{aligned}
$$

where h.c. are hermitian conjugate terms and

$$
\begin{array}{rlrl}
\phi_{n}^{-1} & \equiv \frac{1}{2} \sum_{k} p_{k} \cdot p_{n-k}, & \phi_{n}^{0} \equiv \frac{1}{4} \sum_{k}\left(x_{k} \cdot p_{n-k}+p_{n-k} \cdot x_{k}\right), \\
\phi_{n}^{1} \equiv \frac{1}{2} \sum_{k} x_{k} \cdot x_{n-k}, & \phi_{n}^{L} \equiv-i \frac{1}{2} \sum_{k} k\left(x_{k} \cdot p_{n-k}+p_{n-k} \cdot x_{k}\right),
\end{array}
$$


which are the Fourier modes of the hermitian operator constraints corresponding to (6.1). The BRST charge (6.3) is formally nilpotent and a consistent BRST quantization is possible if there exists a BRST invariant vacuum state on which we have a nilpotent BRST operator. In order to investigate the existence of such a vacuum state we need the extended constraint operators defined by

$$
\begin{aligned}
\tilde{\phi}_{n}^{-1} \equiv\left[\mathcal{Q}, b_{n}^{-1}\right]= & \phi_{n}^{-1}+\sum_{k}\left(2 i c_{k}^{1} b_{n-k}^{0}+i c_{k}^{0} b_{n-k}^{-1}-(n+k) c_{k}^{L} b_{n-k}^{-1}\right) \\
\tilde{\phi}_{n}^{0} \equiv\left[\mathcal{Q}, b_{n}^{0}\right]= & \phi_{n}^{0}+\frac{1}{2} \sum_{k}\left(i c_{k}^{1} b_{n-k}^{1}-i b_{n-k}^{1} c_{k}^{1}-2 n c_{k}^{L} b_{n-k}^{0}-\right. \\
& \left.-i c_{k}^{-1} b_{n-k}^{-1}+i b_{n-k}^{-1} c_{k}^{-1}\right) \\
\tilde{\phi}_{n}^{1} \equiv\left[\mathcal{Q}, b_{n}^{1}\right]= & \phi_{n}^{1}-\sum_{k}\left(2 i c_{k}^{-1} b_{n-k}^{0}+i c_{k}^{0} b_{n-k}^{1}+(n-k) c_{k}^{L} b_{n-k}^{1}\right) \\
\tilde{\phi}_{n}^{L} \equiv\left[\mathcal{Q}, b_{n}^{L}\right]= & \phi_{n}^{L}-\frac{1}{2} \sum_{k}\left((k+n)\left(c_{k}^{-1} b_{n-k}^{-1}-b_{n-k}^{-1} c_{k}^{-1}+c_{k}^{L} b_{n-k}^{L}-b_{n-k}^{L} c_{k}^{L}\right)+\right. \\
& \left.\left.+(k-n)\left(c_{k}^{1} b_{n-k}^{1}-b_{n-k}^{1} c_{k}^{1}\right)+k\left(c_{k}^{0} b_{n-k}^{0}-b_{n-k}^{0} c_{k}^{0}\right)\right]\right) .
\end{aligned}
$$

These operators are shown to satisfy the following commutator algebra

$$
\begin{aligned}
& {\left[\tilde{\phi}_{m}^{1}, \tilde{\phi}_{n}^{-1}\right]=2 i \tilde{\phi}_{m+n}^{0}, \quad\left[\tilde{\phi}_{m}^{L}, \tilde{\phi}_{n}^{L}\right]=(m-n) \tilde{\phi}_{m+n}^{L}, \quad\left[\tilde{\phi}_{m}^{0}, \tilde{\phi}_{n}^{L}\right]=m \tilde{\phi}_{m+n}^{0},} \\
& {\left[\tilde{\phi}_{m}^{1}, \tilde{\phi}_{n}^{0}\right]=i \tilde{\phi}_{m+n}^{1}, \quad\left[\tilde{\phi}_{m}^{-1}, \tilde{\phi}_{n}^{0}\right]=-i \tilde{\phi}_{m+n}^{-1},} \\
& {\left[\tilde{\phi}_{m}^{1}, \tilde{\phi}_{n}^{L}\right]=(m+n) \tilde{\phi}_{m+n}^{1}, \quad\left[\tilde{\phi}_{m}^{-1}, \tilde{\phi}_{n}^{L}\right]=(m-n) \tilde{\phi}_{m+n}^{-1} .}
\end{aligned}
$$

These commutators are non-anomalous as required by the formal nilpotence of $Q$.

Following [4] we consider now a vacuum state which satisfies the conditions

$$
p_{n}^{M}|0\rangle=b_{n}^{-1}|0\rangle=c_{n}^{1}|0\rangle=0, \quad \forall n .
$$

These conditions are consistent with a BRST invariant vacuum state since

$$
\left[Q, p_{n}^{M}\right]|0\rangle=\tilde{\phi}_{n}^{-1}|0\rangle=\left[Q, c_{n}^{1}\right]|0\rangle=0, \quad \forall n,
$$

are satisfied due to (6.7). Now these conditions do not specify a unique vacuum. We need further conditions for that. We may ghost fix the vacuum by the conditions

$$
b_{n}^{0}|0\rangle=b_{n}^{L}|0\rangle=0, \quad \forall n,
$$

Their consistency conditions are

$$
\tilde{\phi}_{n}^{0}|0\rangle=\tilde{\phi}_{n}^{L}|0\rangle=0, \quad \forall n,
$$

and are satisfied for $n \neq 0$ due to (6.7). By means of the regularization (2.7) we find furthermore that

$$
\tilde{\phi}_{0}^{L}|0\rangle=0
$$

for any $s$ due to the symmetry properties we had in (4.9). Using (2.12) we have by a direct calculation

$$
\tilde{\phi}_{0}^{0}|0\rangle=\frac{i}{2}(d-2) \alpha|0\rangle
$$


in the limit $s \rightarrow 0$. Thus, $\tilde{\phi}_{0}^{0}$ has an imaginary continuous spectrum. The above vacuum state is therefore only BRST invariant for $d=2$. (There might exist BRST invariant states built from the above vacuum state. However, we have not found any.)

If we define a new charge like in the bosonic string by

$$
Q^{\prime}=Q-\frac{i}{2}(d-2) \alpha c_{0}^{0}
$$

then the above vacuum state is BRST invariant under the new charge $Q^{\prime}$ for any dimension $d$. However, $Q^{\prime}$ is then neither hermitian nor nilpotent for $d \neq 2$.

In fact, $Q$ is nilpotent on $|0\rangle$ for any dimension $d$. This may be checked as in the previous models, by computing the algebra of the extended contraints acting on the vacuum state. The most non-trivial of these equations read

$$
\left[\tilde{\phi}_{m}^{-1}, \tilde{\phi}_{-m}^{1}\right](s)|0\rangle=\frac{2-d}{2} \sum_{k} f^{(\alpha)}(|k|, s) f^{(\alpha)}(|k+m|, s)|0\rangle .
$$

Here

$$
\begin{aligned}
& \sum_{k} f^{(\alpha)}(|k|, s) f^{(\alpha)}(|k+m|, s)=\zeta\left(s, \alpha+\frac{1}{2}|m|,-\frac{m^{2}}{4}\right)+ \\
& +\zeta\left(s, \alpha-\frac{1}{2}|m|,-\frac{m^{2}}{4}\right)+g(m, s),
\end{aligned}
$$

where we have introduced the zeta function 8

$$
\zeta(s, a, b) \equiv \sum_{k} \frac{1}{\left[(k+a)^{2}+b\right]^{s}}
$$

and $g(m, s)$ involves only finite sums and $g(m, 0)=-1$. Now since (we are indebted to Per Salomonson for this simple argument)

$$
\left.\frac{d \zeta}{d b}\right|_{s=0}=\left.s \sum_{k} \frac{1}{\left[(k+a)^{2}+b\right]^{s+1}}\right|_{s=0}=0
$$

we have

$$
\zeta(0, a, b)=\zeta(0, a, 0)=\zeta(0 \mid a)=\frac{1}{2}-a,
$$

which implies

$$
\left.\sum_{k} f^{(\alpha)}(|k|, s) f^{(\alpha)}(|k+m|, s)\right|_{s=0}=-2 \alpha .
$$

Hence

$$
\left[\tilde{\phi}_{m}^{-1}, \tilde{\phi}_{-m}^{1}\right]|0\rangle=(d-2) \alpha|0\rangle,
$$

which is consistent with $(6.6)$ and $(6.12)$. Commutators of other constraint operators may similarly be shown to consistently act on the vacuum state in accordance with eq (6.6). Thus the nilpotency of the BRST operator holds as a true operator equation in the chosen 
state space. For $d \neq 2$ the considered vacuum state is not BRST invariant. Furthermore, we have not been able to find any BRST invariant state in the state space. If there does not exist BRST invariant states the theory is non-trivial only in $d=2$.

Now instead of the conditions (6.9) we may also ghost fix the vacuum state by the conditions

$$
c_{n}^{0}|0\rangle=b_{n}^{L}|0\rangle=0, \quad \forall n .
$$

In this case all consistency conditions are satisfied, which means that this vacuum is BRST invariant under the original formally nilpotent BRST charge (6.3) for any dimension $d$. Hence, we have found two vacua: one which is BRST invariant only for $d=2$ and another which is BRST invariant for all dimensions. However, since we work in the minimal sector we must make sure that there exists a dual vacuum state $|\overline{0}\rangle$. It is straight-forward to show that $|\overline{0}\rangle$ exists for any dimensions in the first case above but only for $d=2$ in the second case. Thus, both solutions yield equivalent results and we have found a BRST invariant vacuum only for $d=2$ in agreement with the result of [4]. That we have two solutions requires a selection condition. (What we have here is a noncanonical situation in the language of [5].)

\section{Alternative quantizations of the bosonic conformal string}

We will here consider two alternative set of state spaces to the one treated in the previous section.

\subsection{A consistent solution at $d=4$}

First we consider a state space with a vacuum state defined through the conditions

$$
p_{n}^{\mu}|0\rangle=p_{n}^{+}|0\rangle=x_{n}^{+}|0\rangle=b_{n}^{-1}|0\rangle=c_{n}^{1}|0\rangle=b_{n}^{0}|0\rangle=b_{n}^{L}|0\rangle=0, \quad \forall n .
$$

It is easily checked that this vacuum state satisfies

$$
\tilde{\phi}_{n}^{-1}|0\rangle=\tilde{\phi}_{n}^{L}|0\rangle=0, \quad \forall n .
$$

Furthermore,

$$
\tilde{\phi}_{0}^{0}|0\rangle=\frac{i}{2}(d-4) \alpha|0\rangle
$$

in the limit $s \rightarrow 0$. By the same reasoning as in the previous section this leads to the conclusion that the above vacuum state is only BRST invariant for $d=4$. We must also check the closure of the extended constraints when acting on the vacuum state. In precisely the same way that lead to eq. $(6.20)$ one finds in this case

$$
\left[\tilde{\phi}_{m}^{-1}, \tilde{\phi}_{-m}^{1}\right]|0\rangle=(d-4) \alpha|0\rangle
$$

which is consistent with (6.6) and (7.3). Other commutators of constraint operators may similarly be shown to consistently act on the vacuum state. Concluding, we have a nonanomalous theory for any $d$ and the vacuum state is BRST invariant for $d=4$. We have 
not been able to find BRST invariant states for other $d$. The vacuum dual to $|0\rangle$ is defined by

$$
x_{n}^{\mu}|\overline{0}\rangle=x_{n}^{-}|\overline{0}\rangle=p_{n}^{-}|\overline{0}\rangle=c_{n}^{-1}|\overline{0}\rangle=b_{n}^{1}|\overline{0}\rangle=c_{n}^{0}|\overline{0}\rangle=c_{n}^{L}|\overline{0}\rangle=0, \quad \forall n .
$$

It is easily shown that this vacuum is BRST invariant for any $d$.

The state space that we have defined here treats the $x^{ \pm}$coordinates differently than the $x^{\mu}$ coordinates. Thus we have lost manifest $d+2$ dimensional $S O(d, 2)$ covariance. However, as the $x^{\mu}$ coordinates are regarded as the physical ones (in the sense of the gauge fixing conditions $(\sqrt[6.2]{ })$ ), we still have manifest Lorentz covariance in this physical subspace. (If we do not insist on manifest Lorentz covariance, we may treat $x^{0}$ and one of the space coordinates in a similar fashion as $x^{ \pm}$and get a BRST invariant vacuum in $d=6$.)

\subsection{An inconsistent solution}

We now consider another choice of state space. In analogy with the alternative treatment of the tensionless string in section 5 we may for the conformal string try a vacuum state satisfying

$$
p_{m}^{M}|0\rangle=x_{m}^{M}|0\rangle=0, \quad m>0 .
$$

This alternative quantization was investigated in [3]. The consistency conditions to (7.6) requires

$$
c_{m}^{-1}|0\rangle=c_{m}^{0}|0\rangle=c_{m}^{1}|0\rangle=c_{m}^{L}|0\rangle=0, \quad m>0 .
$$

The conditions (7.6) and (7.7) allow for a ghost fixing of the form

$$
\begin{gathered}
c_{0}^{1}|0\rangle=0, \quad b_{m}^{1}|0\rangle=0, \quad m>0, \\
b_{m}^{-1}|0\rangle=b_{m}^{0}|0\rangle=b_{m}^{L}|0\rangle=0, \quad m \geq 0 .
\end{gathered}
$$

The consistency conditions for (7.8) are automatically satisfied as well as those of (7.9) for $m>0$. However,

$$
\tilde{\phi}_{0}^{-1}|0\rangle=0
$$

and

$$
\tilde{\phi}_{0}^{0}|0\rangle=0
$$

yields further conditions on the vacuum state. They may be satisfied. (Eq.(7.11) fixes the conformal dimension of the vacuum state.) The problem is the last one which yields

$$
\tilde{\phi}_{0}^{L}|0\rangle=(d-2)\left(\frac{1}{2} \alpha^{2}-\frac{1}{12}\right)|0\rangle .
$$

Checking the commutation relations we find

$$
\lim _{s \rightarrow 0}\left[\tilde{\phi}_{m}^{L}, \tilde{\phi}_{-m}^{L}\right](s)=2 m \tilde{\phi}_{0}^{L}+\frac{1}{6}(d-26) m^{3}-m(d-2) \alpha^{2},
$$


where $\tilde{\phi}_{0}^{L}$ on the right-hand side is the normal ordered operator in (7.12). Notice that (7.13) coincide almost exactly with (5.11) although we here have $d+2$ coordinates and 4 ghost fields. It looks now as if the situation is the same as for the tensionless string in section 5, i.e. it looks as if we may follow the arguments after (5.11) leading to a consistent BRST quantization in $d=26$. However, for the conformal string there is an additional nontrivial commutator for which we find

$$
\lim _{s \rightarrow 0}\left[\tilde{\phi}_{m}^{1}, \tilde{\phi}_{-m}^{-1}\right](s)|0\rangle=\left(2 i \tilde{\phi}_{0}^{0}-\frac{1}{2}(d-6) m\right)|0\rangle,
$$

where $\tilde{\phi}_{0}^{0}$ on the right-hand side is the normal ordered operator that follows from (6.5) through our regularization, i.e.

$$
\begin{aligned}
& \tilde{\phi}_{0}^{0}=\frac{1}{4}\left(x_{0} p_{0}+p_{0} x_{0}\right)+\frac{1}{2} \sum_{k=1}^{\infty}\left(p_{-k} \cdot x_{k}+x_{-k} \cdot p_{k}\right)+i\left(c_{0}^{1} b_{0}^{1}-c_{0}^{-1} b_{0}^{-1}\right)+ \\
& +i \sum_{k=1}^{\infty}\left(b_{-k}^{-1} \cdot c_{k}^{-1}-c_{-k}^{-1} \cdot b_{k}^{-1}-b_{-k}^{1} \cdot c_{k}^{1}+c_{-k}^{1} \cdot b_{k}^{1}\right) .
\end{aligned}
$$

The relation (7.14) says that $Q$ can only be nilpotent in $d=6$ which contradicts the above result that required $d=26$. It follows that there is no consistent BRST quantization of the conformal string on the vacuum considered here. This result agrees with [3].

\section{Alternative state space for the bosonic string?}

In view of the treatments in sections 4 and 5 one may wonder if one may not have a corresponding vacuum state also in the ordinary bosonic string case. Below we demonstrate that this is not the case. The classical constraints (3.1) may also be written as

$$
P^{\mu}(\sigma) P_{\mu}(\sigma)+T^{2} X^{\mu \prime}(\sigma) X_{\mu}^{\prime}(\sigma)=0, \quad P^{\mu}(\sigma) X_{\mu}^{\prime}(\sigma)=0 .
$$

The Fourier modes of the corresponding hermitian constraint operators are

$$
\begin{aligned}
\Phi_{n}^{-1} & \equiv \frac{1}{2} \sum_{k} p_{k} \cdot p_{n-k}-2 T^{2} \sum_{k} k(n-k) x_{k} \cdot x_{n-k} \\
\phi_{n}^{L} & \equiv-i \frac{1}{2} \sum_{k} k\left(x_{k} \cdot p_{n-k}+p_{n-k} \cdot x_{k}\right) .
\end{aligned}
$$

The hermitian and formally nilpotent BRST charge (3.5) may be rewritten as

$$
\begin{aligned}
Q= & \sum_{k}\left(\Phi_{-k}^{-1} c_{k}^{-1}+\phi_{-k}^{L} c_{k}^{L}\right)- \\
& -\frac{1}{2} \sum_{k, l}(k-l)\left(c_{-k}^{-1} c_{-l}^{L} b_{k+l}^{-1}+\frac{1}{2} c_{-k}^{L} c_{-l}^{L} b_{k+l}^{L}+4 T^{2} c_{-k}^{-1} c_{-l}^{-1} b_{k+l}^{L}+\right. \\
& \left.+b_{k+l}^{-1} c_{-k}^{-1} c_{-l}^{L}+\frac{1}{2} b_{k+l}^{L} c_{-k}^{L} c_{-l}^{L}\right) .
\end{aligned}
$$

(Notice that the ghost variables $c_{k}^{L}, b_{k}^{L}$ here are not the same as in (3.5).) The extended constraints are here given by

$$
\begin{aligned}
\tilde{\phi}_{n}^{L} & \equiv\left[Q, b_{n}^{L}\right]=\phi_{n}^{L}-\frac{1}{2} \sum_{k}(n+k)\left(c_{k}^{-1} b_{n-k}^{-1}-b_{n-k}^{-1} c_{k}^{-1}+c_{k}^{L} b_{n-k}^{L}-b_{n-k}^{L} c_{k}^{L}\right), \\
\tilde{\Phi}_{n}^{-1} & \equiv\left[Q, b_{n}^{-1}\right]=\Phi_{n}^{-1}-\sum_{k}(n+k)\left(c_{k}^{L} b_{n-k}^{-1}+4 T^{2} c_{k}^{-1} b_{n-k}^{L}\right),
\end{aligned}
$$


A direct calculation of the commutator $\left[\tilde{\phi}_{m}^{L}, \tilde{\phi}_{-m}^{L}\right]$ gives

$$
\begin{aligned}
& {\left[\tilde{\phi}_{m}^{L}, \tilde{\phi}_{-m}^{L}\right](s)=} \\
& m \sum_{k}\left(-i k x_{k} \cdot p_{-k}-k c_{k}^{L} b_{-k}^{L}-k c_{k}^{-1} b_{-k}^{-1}\right)\left(f^{(\alpha)}(|m+k|, s)+f^{(\alpha)}(|m-k|, s)\right)+ \\
& +\sum_{k}\left(-i k^{2} x_{k} \cdot p_{-k}+\left(2 m^{2}-k^{2}\right)\left(c_{k}^{L} b_{-k}^{L}+c_{k}^{-1} b_{-k}^{-1}\right)\right)\left(f^{(\alpha)}(|m+k|, s)-f^{(\alpha)}(|m-k|, s)\right) .
\end{aligned}
$$

The $s \rightarrow 0$ limit yields

$$
\left[\tilde{\phi}_{m}^{L}, \tilde{\phi}_{-m}^{L}\right]=2 m \tilde{\phi}_{0}^{L}
$$

which is consistent with the fact that the BRST charge (8.3) is nilpotent. One may note here that the second line in the last relation goes to zero as $s$ goes to zero if the operator

$$
\Psi_{k, m} \equiv-i k^{2} x_{k} \cdot p_{-k}+\left(2 m^{2}-k^{2}\right)\left(c_{k}^{L} b_{-k}^{L}+c_{k}^{-1} b_{-k}^{-1}\right)
$$

is well-defined. This is true for a state space where the vacuum has the form of the tensionless string vacuum defined by the condition

$$
p_{n}^{\mu}|0\rangle=0, \quad \forall n .
$$

However this vacuum state is not appropriate for the tensile bosonic string. Consistency conditions of the form

$$
\left[Q, p_{n}^{\mu}\right]|0\rangle=0, \quad \forall n
$$

would require the ghost part of such a state to satisfy the relations

$$
c_{n}^{-1}|0\rangle=0, \quad \forall n
$$

for which the consistency conditions,

$$
\left[Q, c_{n}^{-1}\right]|0\rangle=0, \quad \forall n
$$

are satisfied. In fact, it is possible to ghost fix this vacuum state in a BRST invariant way using the original BRST charge which then also is nilpotent for any dimension $d$. However, there exists no dual vacuum state, $|\overline{0}\rangle$, to this solution. Note that $|\overline{0}\rangle$ must satisfy

$$
x_{n}^{\mu}|\overline{0}\rangle=b_{n}^{-1}|\overline{0}\rangle=0
$$

for which the consistency condition

$$
\left[Q, b_{n}^{-1}\right]|\overline{0}\rangle=0
$$

cannot be satisfied. 


\section{Alternatives with negative norm states.}

In the alternative treatments in section 5 and 7 we considered a vacuum state of the type

$$
p_{m}^{\mu}|0\rangle=x_{m}^{\mu}|0\rangle=0, \quad m>0 .
$$

It lead to a "consistent" BRST quantization for the bosonic tensionless string in $d=26$. In fact, even the ordinary bosonic string has a vacuum state satisfying (9.1). To see this one may notice that (9.1) is equivalent to (We let $|0\rangle$ be an eigenstate of $p_{0}^{\mu}$, i.e. we set $|0\rangle=|p\rangle$.)

$$
\alpha_{m}^{\mu}|p\rangle=0, \quad \tilde{\alpha}_{-m}^{\mu}|p\rangle=0, \quad m>0 .
$$

due to the relations (3.3). Now (9.2) is different from the standard vacuum (3.10). The consistency conditions of the latter condition requires

$$
c_{-m}^{K}|p\rangle=0, \quad m>0
$$

which means that the vacuum may be ghost fixed by

$$
b_{-m}^{K}|p\rangle=0, \quad m \geq 0
$$

together with

$$
c_{m}^{L}|p\rangle=0, \quad m>0 ; \quad b_{m}^{L}|p\rangle=0, \quad m \geq 0 .
$$

Calculating commutators for finite $s$ and then taking the limit $s \rightarrow 0$ we find

$$
\left[\tilde{K}_{m}, \tilde{K}_{-m}\right]=2 m \tilde{K}_{0}-\frac{1}{12}(d-26) m^{3}+\frac{1}{2} m(d-2) \alpha^{2}
$$

where

$$
\tilde{K}_{0}=\frac{1}{2} \alpha_{0}^{2}+\sum_{k=1}^{\infty}\left(\tilde{\alpha}_{k} \cdot \tilde{\alpha}_{-k}-k b_{k}^{K} c_{-k}^{K}-k c_{k}^{K} b_{-k}^{K}\right)-\frac{1}{2}(d-2)\left(\frac{1}{2} \alpha^{2}-\frac{1}{12}\right) .
$$

We may then define a new BRST charge by

$$
Q^{\prime}=Q-\frac{d-2}{4} \alpha^{2}\left(c_{0}^{L}-c_{0}^{K}\right),
$$

which then is nilpotent for $d=26$. In $d=26$ the new extended constraints are

$$
\begin{aligned}
& \tilde{L}_{0}^{\prime}=\frac{1}{2} \alpha_{0}^{2}+\sum_{k=1}^{\infty}\left(\alpha_{-k} \cdot \alpha_{k}+k b_{-k}^{L} c_{k}^{L}+k c_{-k}^{L} b_{k}^{L}\right)-1, \\
& \tilde{K}_{0}^{\prime}=\frac{1}{2} \alpha_{0}^{2}+\sum_{k=1}^{\infty}\left(\tilde{\alpha}_{k} \cdot \tilde{\alpha}_{-k}-k b_{k}^{K} c_{-k}^{K}-k c_{k}^{K} b_{-k}^{K}\right)+1,
\end{aligned}
$$

However, $\tilde{L}_{0}^{\prime}|p\rangle=0$ requires $p^{2}=8 T$ while $\tilde{K}_{0}^{\prime}|p\rangle=0$ requires $p^{2}=-8 T$. It follows that neither of these vacua are BRST invariant, or in other words we have no BRST invariant vacuum satisfying (9.1). Still there are BRST invariant states and they are massless with spin 0,1 , and 2 , and spinless with $p^{2}= \pm 8 T$. Note, however, that since $\tilde{\alpha}_{m}^{\mu}, m>0$, act as creation operators the space components $\tilde{\alpha}_{m}^{i}$ yield negative normed states. Thus, the BRST invariant state space have negatively normed states. The same feature was also found for the alternative treatment of the tensionless string in section 5. (This alternative treatment has only massless states [16].) It is clear that the existence of indefinite metric states in the BRST invariant sector makes both these models inconsistent. 
Acknowledgments P.S. wish to thank R. vonUnge and U. Lindström for useful comments and suggestions. The work of P.S. was supported by the European Commission TMR Programme under the grant ERBFMBI-CT97-2823.

\section{References}

[1] M. Kato and K. Ogawa, Nucl. Phys. B212, 443 (1983)

S. Hwang, Phys. Rev. D28, 2614 (1983)

[2] D. Friedan, E. Martinec and S. Shenker, Nucl. Phys. B271, 93 (1986)

[3] J. Isberg, "Tensionless Strings with Manifest Space-Time Conformal Invariance" USITP-92-10 (1992)

[4] H. Gustafsson, U. Lindström, P. Saltsidis, B. Sundborg and R.v. Unge, Nucl. Phys. B440 (1995) 495.

[5] S. Hwang and R. Marnelius, Nucl. Phys. B315, 638 (1989); ibid. B320, 476 (1989)

[6] S. Hwang, Nucl. Phys. B322, 107 (1989)

R. Marnelius, Nucl. Phys. B372, 218 (1992); B384, 318 (1992)

[7] I. A. Batalin and E. S. Fradkin, Ann. Inst. Henri Poincaré 49, 145-214 (1988)

[8] E. Elizalde et al, Zeta Regularization Techniques with Applications, World Scientific Pub. Co.. (1994)

[9] A. Schild, Phys. Rev. D16 (1977) 1722.

A. Karlhede and U. Lindström, Class. Quant. Grav. 3 (1986) L73.

F. Lizzi, B. Rai, G. Sparano and A. Srivastava, Phys.Lett. 182B (1986) 326.

R. Amorim and J. Barcelos-Neto, Z.Phys.C38 (1988) 643.

U. Lindström, B. Sundborg and G. Theodoridis, Phys. Lett. 253B (1991) 319.

U. Lindström, B. Sundborg and G. Theodoridis, Phys. Lett. 258B (1991) 331.

[10] F. Lizzi, B. Rai, G. Sparano and A. Srivastava, Phys.Lett. 182B (1986) 326.

J. Isberg, U. Lindström, B. Sundborg and G. Theodoridis, Nucl. Phys., B411 (1994) 122.

[11] J. Gamboa, C. Ramirez, and M. Ruiz-Atlaba, Phys. Lett. 225B, 335 (1989)

[12] R. Marnelius, Nucl. Phys. B412, 817 (1994),

I. A. Batalin and R. Marnelius, Nucl. Phys. B442, 669 (1995)

[13] P. Saltsidis, Nucl. Phys. B446 (1995) 286

[14] P. Saltsidis, Phys. Lett. 401B (1997) 21

[15] E. T. Whittaker and G. N. Watson, Modern Analysis. (fourth edition), Cambridge University Press. (1952)

[16] J. Gamboa, C. Ramirez, and M. Ruiz-Atlaba, Nucl. Phys. B338, 143 (1990) 
[17] R. Marnelius, Phys. Rev. D20, 2091 (1979),

R. Marnelius and B. Nilsson, Phys. Rev. D22, 830 (1979) 